\title{
Comparison of modified Woo's solar phase scintillation model with ESA's BepiColombo superior solar conjunction measurement data for X-band and Ka-band
}

\author{
Shun-Ping Chen ${ }^{1,2}$ [D Jose Villalvilla ${ }^{2}$
}

Received: 6 October 2021 / Revised: 16 January 2022 / Accepted: 24 January 2022 / Published online: 24 February 2022

(c) The Author(s) 2022

\begin{abstract}
Solar phase scintillation is of major interest in deep space mission operations for designing a communications system capable of transmitting signals when the path is close to the Sun, but also for improving the navigation under these conditions. The basis of this work is Woo's solar phase scintillation theory which reported a useful model for the spectral power density of phase scintillations of a signal traveling through a medium close to the Sun. In this paper, Woo's solar phase scintillation formula is used for the simulation of the spectral density of phase fluctuations induced by the Sun on coherent signals (uplink and downlink carriers are coherently related by a fractional ratio implemented by the spacecraft's transponder), for the two downlink frequency bands supported by the ESA deep space mission BepiColombo. In particular, the model has been compared to measurement data collected at low Sun Earth Probe (SEP) angles during the superior solar conjunction campaign in March 2021 (in its cruise phase to Mercury). The comparison shows a good match between the phase noise spectral densities of the modified formula and measurement data, therefore, confirming that the formula can be used for the planning and verification of the future near Sun measurements.
\end{abstract}

Keywords Deep space communications $\cdot$ Phase noise $\cdot$ Solar wind $\cdot$ Solar plasma $\cdot$ Phase scintillation $\cdot$ Scattering $\cdot$ Solar superior conjunction $\cdot$ SEP Sun earth probe angle $\cdot$ BepiColombo

\section{Introduction}

In the past decades, deep space communication technologies have been developed continuously, both in the radio frequency bands and in the infrared spectrum for laser transmissions. Due to the solar wind activities and the ionized solar plasma, a radio transmission suffers phase and amplitude scintillation, as well as spectral broadening for radio paths close to the Sun with a heliocentric distance of $2 R_{\mathrm{s}}-215 R_{\mathrm{s}}$ where $R_{\mathrm{S}}$ is the Sun radius. The intensity of the solar plasma effects depends on the electron densities and

Shun-Ping Chen-Visiting Scientist at ESA / ESOC

Shun-Ping Chen

shun-ping.chen@h-da.de

1 Institute of Communication Technologies, Darmstadt University of Applied Sciences, Birkenweg 8, 64295 Darmstadt, Germany

2 ESA/ESOC European Space Agency, Operations Centre, Robert-Bosch-Straße 5, 64293 Darmstadt, Germany wave number as well as the velocities of the solar wind, and were investigated in detail in [1-3]. The phase scintillation spectral density distribution, by taking all these factors into account, has proven to be a very useful method to characterize radio scattering measurement results. The better understanding of the solar plasma effects on the scattering of radio waves is essential for communications' design and spacecraft's navigation in interplanetary missions.

The motivation of this work is to verify the formula first published in [1-3] for S-band, and further modified for a coherent two-way mode, leading to a very useful formula to compute the phase scintillations' dependence on the Sun Earth Probe (SEP) angles when operating in coherent/non coherent transponder mode, generally for S-band, X-band, and Ka-band near the Sun [1, 4, 5]. In Fig. 1 the constellation of Sun, Earth and Probe (Spacecraft) with SEP is illustrated schematically.

The original formula of Woo was based on the electron density spectra's dependence on wave number, solar wind velocity, and heliocentric distance to the line-of-sight path. The phase scintillation formula was derived and compared 
Fig. 1 Schematic constellation of Sun, Earth, Probe and SEP angle
Sun radius $R_{S}=695,500 \mathrm{~km}$ or $0.00465 \mathrm{AU}$

Distance Sun-Earth $\mathrm{R}_{\mathrm{ES}}=149,597,871 \mathrm{~km}$ or $1 \mathrm{AU}$

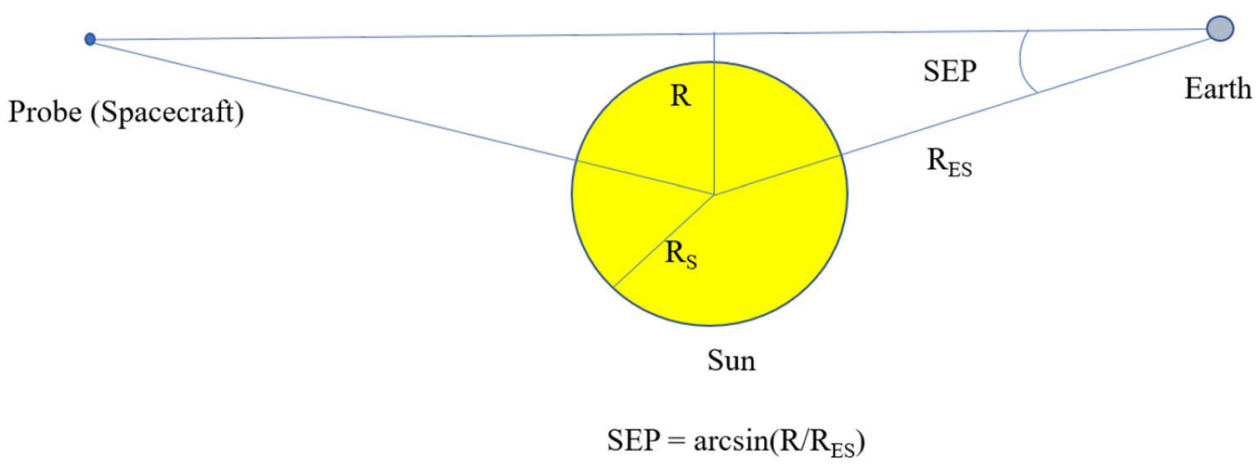

with the S-band measurement results [2] collected during the Viking missions I and II between 1976-1978.

On one hand Woo's formula complied to the Kolmogorov turbulence theory, on the other hand the simulation results, by using this formula, matched pretty well to the S-band phase scintillation measurement data collected by NASA JPL during the Viking missions.

A modified formula, scaled to other carrier frequencies, has been derived and further modified to be applied to coherent single-frequency links [1, 4-6]. By using this modified formula, the phase shift spectral densities of X-band and Ka-band for SEP angles $5.1^{\circ}, 3.6^{\circ}, 2.9^{\circ}$, and $1.2^{\circ}$ are calculated in this work, and are compared with the corresponding measurement data collected during the ESA BepiColombo mission [7]. BepiColombo is an ESA mission to Mercury, launched in October, 2018 and arrival to Mercury planned in late 2025. During its cruise phase, open loop data were collected by 35 meter ESA Deep Space Antennae in Malargüe (Argentina) and Cebreros (Spain) over several days during the superior solar conjunction, covering SEP angles from $1.2^{\circ}$ to $5.1^{\circ}$, a region which is very critical for radio frequency transmissions due to the amplitude and phase scintillations, and therefore of great interest to space science and deep space communications and navigation.

The relatively good match between the formula and the measurement data confirms that the modified formula can be used for mission planning, and for verification of the future radio frequency measurement results not only in S-band, but also in X-band and Ka-band.

\section{Theoretical background}

For the one-way downlink operation (non-coherent mode), Woo first derived the formula for the single-frequency phase scintillation spectral density [2, 3] from the numerically integrated model for differential phase as
$W_{\Phi}(f)=\frac{W_{\Phi \mathrm{d}}(f)}{0.857}=6.534 \cdot f^{-2.65} \cdot\left(\frac{R}{R_{\mathrm{S}}}\right)^{-2.45}\left[\frac{\mathrm{rad}^{2}}{\mathrm{~Hz}}\right]$

$W_{\Phi \mathrm{d}}(f)$ being the differential phase scintillation spectral density in the dual-frequency case [2].

The received signal at the ground station before the phase locked loop (PLL) is generally characterized by the spectral density of the solar phase noise. To apply the formula to the ESA systems and measurements, the formula was derived and scaled for different carrier frequencies $f_{\mathrm{c}}$ according to [2, 3] for calculating the spectral densities of the phase scintillations as

$S_{\Phi R}(f)=\left(\frac{2.3}{f_{\mathrm{c}}}\right)^{2} \cdot 6.534 \cdot f^{-2.65} \cdot\left(\frac{R}{R_{\mathrm{s}}}\right)^{-2.45}\left[\frac{\mathrm{rad}^{2}}{\mathrm{~Hz}}\right]$

$f_{c}$ in $\mathrm{GHz}$ is the carrier frequency in S-band, X-band (8.42 $\mathrm{GHz}$ ) or $\mathrm{Ka}$-band $(32 \mathrm{GHz}), \mathrm{f}$ in $\mathrm{Hz}$ is the frequency offset, $R$ is the transverse distance from Sun's centre to the connecting line between the spacecraft and Earth, the distance $R_{\mathrm{ES}}$ between Sun and Earth is $149,597,871 \mathrm{~km}$ or 1 $\mathrm{AU}$ (astronomic unit), $R_{s}$ is the radius of the Sun $(695,500$ $\mathrm{km}$ or $0.00465 \mathrm{AU})$. The Sun-Earth Probe angle (SEP) will then be written as

$\mathrm{SEP}=\arcsin \left(\frac{R}{R_{\mathrm{ES}}}\right)$.

In case of coherent two-way operations, the ground station transmits an uplink signal which is received on-board and used to coherently synthesize the downlink carrier frequency. An additional factor is added to the equation (2) to account for the effect of the phase fluctuations on the uplink which are subsequently translated to the downlink according to the turn-around ratio $\mathrm{G}(880 / 749$ in case of $\mathrm{X} / \mathrm{X}, 3344 / 749$ in $\mathrm{X} / \mathrm{Ka}$ ), resulting in the following formula: 
$S_{\Phi R}(f)=\left(\frac{2.3}{f_{\mathrm{c}}}\right)^{2} \cdot 6.534 \cdot f^{-2.65} \cdot\left(\frac{R}{R_{\mathrm{s}}}\right)^{-2.45} \cdot\left(1+G^{4}\right)\left[\frac{\mathrm{rad}^{2}}{\mathrm{~Hz}}\right]$

In radio frequency measurements, not only the pure phase scintillations, but also the thermal noise $S_{\mathrm{tn}}(f)$ deduced from the signal-to-noise ratio are measured, so that the total phase noise spectral density can be derived from $[1,2,6]$

$S_{\Phi R, \text { total }}(f)=S_{\Phi R}(f)+S_{\mathrm{tn}}(f)=S_{\Phi R}(f)+\frac{1}{P_{\mathrm{c}} / N_{0}}$

$P_{\mathrm{c}} / N_{0}$ being the carrier-to-noise spectral density ratio. This value varied from of $45-51 \mathrm{dBHz}$ during the measurement campaign for both frequency bands depending on the link configuration and weather conditions at each moment, leading to a noise floor in the measurements clearly visible at spectral frequencies higher than $10 \mathrm{~Hz}$.

The power spectral density is then estimated by using the Burg's method, an autoregressive approach implemented by Matlab algorithm [8]. The variance of the phase scintillations $\sigma_{\Phi}^{2}$ can be estimated by

$\sigma_{\Phi}^{2}=\int_{f_{\mathrm{b}}}^{f_{\mathrm{u}}} S_{\Phi R}(f) \cdot \mathrm{d} f$

The integral should be calculated from a properly chosen bottom limit $f_{\mathrm{b}}$, basically determined by the total number of the samples and the sampling rate, to an upper limit $f_{\mathrm{u}}$, i.e. from $3 \times 10^{-3} \mathrm{~Hz}$ to $10^{2} \mathrm{~Hz}$ for $\mathrm{X}$-band and Ka-band correspondingly.

\section{Spectral densities for S-band [3]}

In [3] the spectral density of the S-band phase noise scintillation was measured between $0.58^{\circ} \leq \mathrm{SEP} \leq 131.7^{\circ}$. Since the formula (1) for the calculation of spectral density functions [3] is only valid for S-band and SEP angles $5.3^{\circ}-27.7^{\circ}$, we calculated the spectral density functions by using the formula for these angles (Fig. 2), just to compare the above formula (1) and the measurement results of [3] (Fig. 3).

For small SEP angles $0.58^{\circ}$ and $2.8^{\circ}$, the spectral densities derived from the measurement data are slightly higher for the higher spectral frequencies $10^{0}-10^{2} \mathrm{~Hz}$ than the simulation results by using formula (1). Also, due to the thermal noise, Woo calculated the spectral densities for the two-way measurement data only up to $10^{-2}-10^{-1} \mathrm{~Hz}[3]$ for $\mathrm{SEP}=25.5^{\circ}, 131.1^{\circ}$ and $175.25^{\circ}$ (Fig. 3).

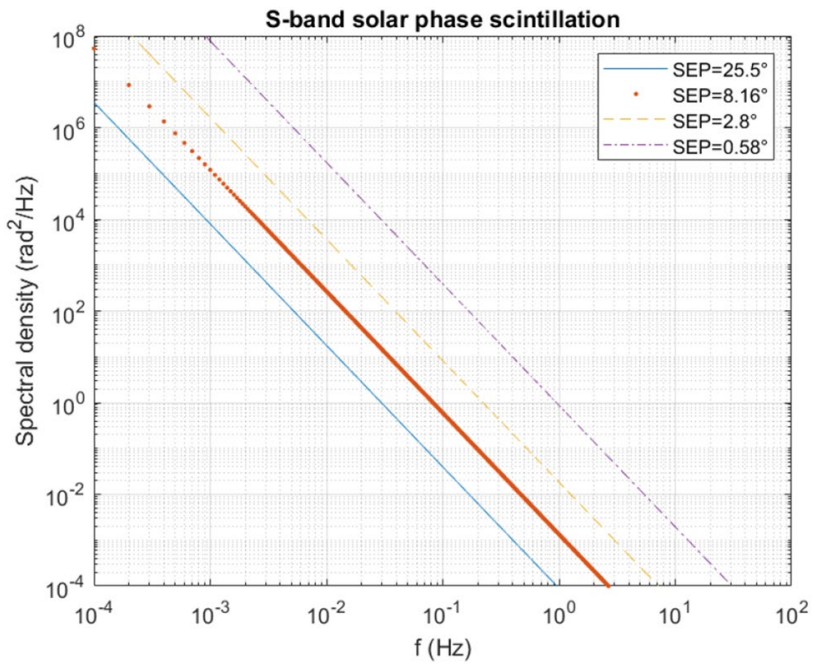

Fig. 2 Spectral density of the S-band calculated by using formula (1) [3]

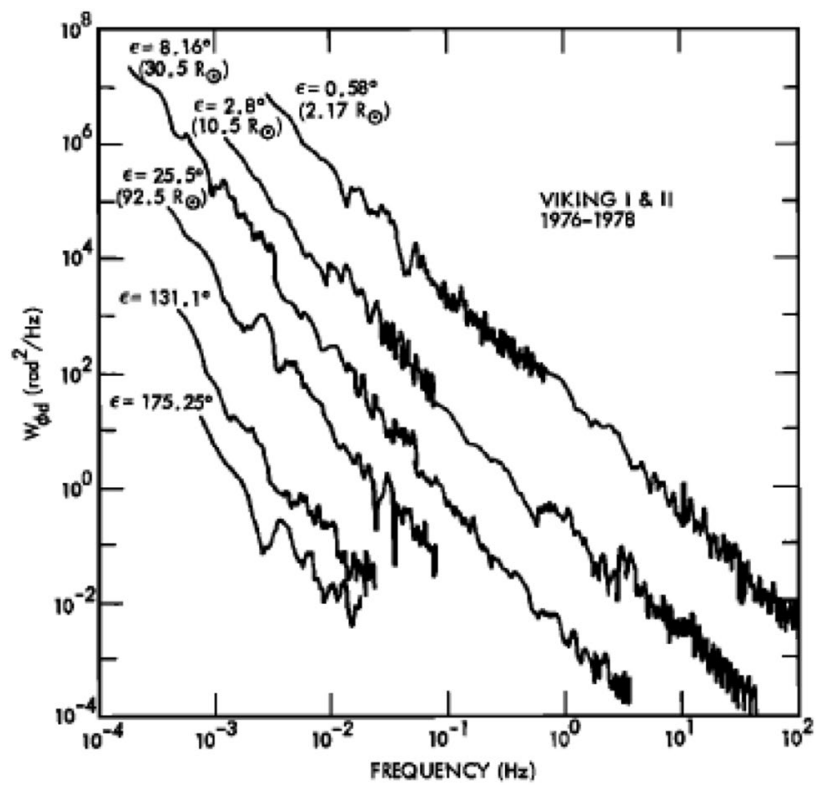

Fig. 3 Original spectral density figure of the S-band measurement results of [3]

\section{Spectral densities for X-band and Ka-band in comparison with ESA BepiColombo superior solar conjunction measurements}

In March 2021, during the cruise phase of the ESA's BepiColombo mission to Mercury, valuable measurement data were collected during several days of the superior solar conjunction at SEP angles that varied from $1.2^{\circ}$ to $5.1^{\circ}$ at the slant range of $1.51 \mathrm{AU}$ for the coherent $\mathrm{X} / \mathrm{X}$-band and 
X/Ka-band downlink. These data [7] are derived from the spacecraft signals down-converted, filtered and sampled to obtain the complex waveform at baseband.

The Doppler effect is compensated in the process by removing the phase trajectory deduced from the orbital predictions computed by Flight Dynamics at ESOC (European Space Operations Centre in Darmstadt, Germany). This Doppler compensation includes a small systematic residual error which is not negligible for the purposes of this work and does not bring any useful information for the present analysis, thus it needs to be removed before any spectral analysis.

It is out of the scope of this work to provide an assessment of this error. The systematic error is estimated and removed from the complex data thanks to the a-posteriori knowledge of the phase, by using a third order polynomial fit.

The phase of the signal is then estimated by computing the argument of the complex data and used as input to the power spectral density (PSD) estimation algorithm. The results are used to compare the phase scintillation spectral densities at different SEP angles with the modified Woo's formula.

The data used for the present analysis were selected among the continuously collected measurement data during several days of campaign, carefully avoiding data at low elevation or unstable weather conditions to avoid any potential impact of the troposphere contribution to the results, which, in most of the cases, should be negligible compared to the contribution of solar plasma. The sampling rate for the measurement data is $2000 \mathrm{samples} / \mathrm{sec}$. A total of 600,000 samples or 300 seconds are taken into account for the spectral density calculations which were performed using the Matlab power spectral density algorithm based on the Burg method among many other different PSD estimation algorithms [8].

The phase scintillations decrease rapidly towards higher spectral frequencies, i.e. $f=10-500 \mathrm{~Hz}$, even below the thermal noise floor at around $10^{-5} \mathrm{rad}^{2} / \mathrm{Hz}$ or approximately $-50 \mathrm{~dB} / \mathrm{Hz}$.

In Fig. 4, the time series of the phase shifts are displayed to show the fluctuations caused by the solar scintillation at SEP angle $5.1^{\circ}$ for Ka-band measurements, whereas the time series of the phase shifts at $1.2^{\circ}$ for Ka-band during the 300 seconds of measurement data are shown in Fig. 5. It can be recognized clearly that the phase scintillation at SEP angle $5.1^{\circ}$ (between -17 radians and +18 radians) is weaker than at the SEP angle $1.2^{\circ}$ (between -130 radians and +100 radians), which can be explained by a reduction of the effect of solar plasma on the signal.

The phase shifts for X-band for different SEP angles are determined in the same procedure as for Ka-band.

These and other phase shifts for X-band and Ka-band for different SEP angles are used for calculating phase

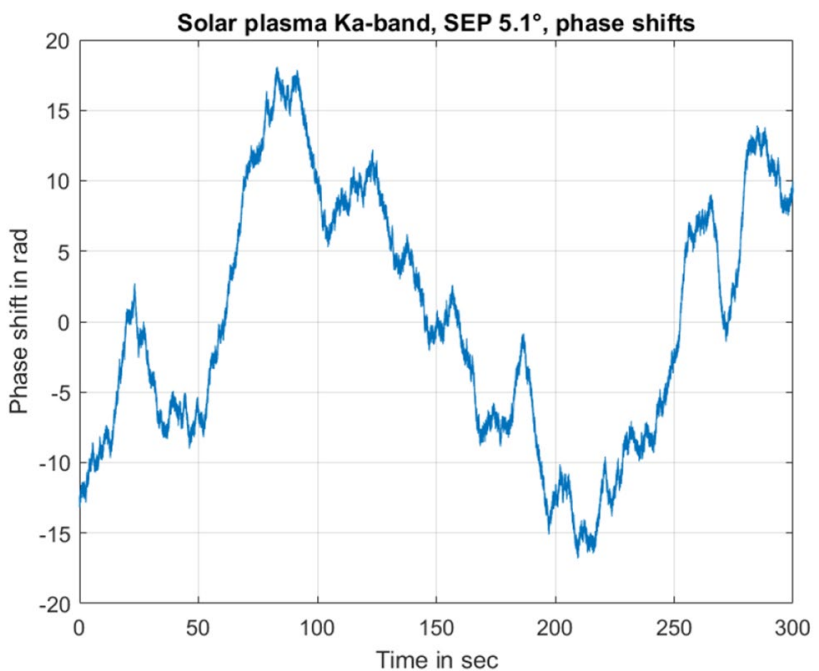

Fig. 4 Phase shifts of the Ka-band measurement data with SEP 5.1 ${ }^{\circ}$

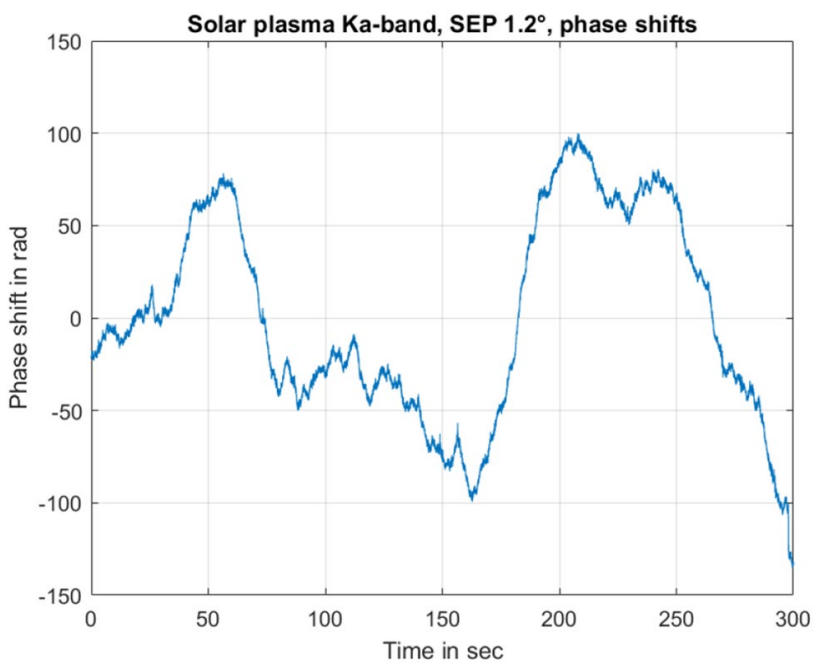

Fig. 5 Phase shifts of the Ka-band measurement data with SEP $1.2^{\circ}$

scintillation spectral densities using the above-mentioned power spectral density algorithms. The phase scintillation spectral densities for SEP angles derived from the measurement data for X-band are shown in Figs. 6, 7, 8 and 9. The simulated phase scintillation spectral densities for SEP $=$ $1.2^{\circ}, 2.9^{\circ}, 3.6^{\circ}$, and $5.1^{\circ}$, by using the modified Woo's formula, and by considering the scaling factor for the $\mathrm{X}$-band carrier frequency as well as the turn-around-factor $\mathrm{G}$ for the coherent operations, are displayed with dashed lines in the diagrams correspondingly.

Figures 6, 7, 8 and 9 show the phase scintillation spectral densities for X-band for the different SEP angles $5.1^{\circ}, 3.6^{\circ}$ , $2.9^{\circ}$ and $1.2^{\circ}$ calculated following the procedure described above. Figures 10, 11, 12 and 13 show the phase scintillation 


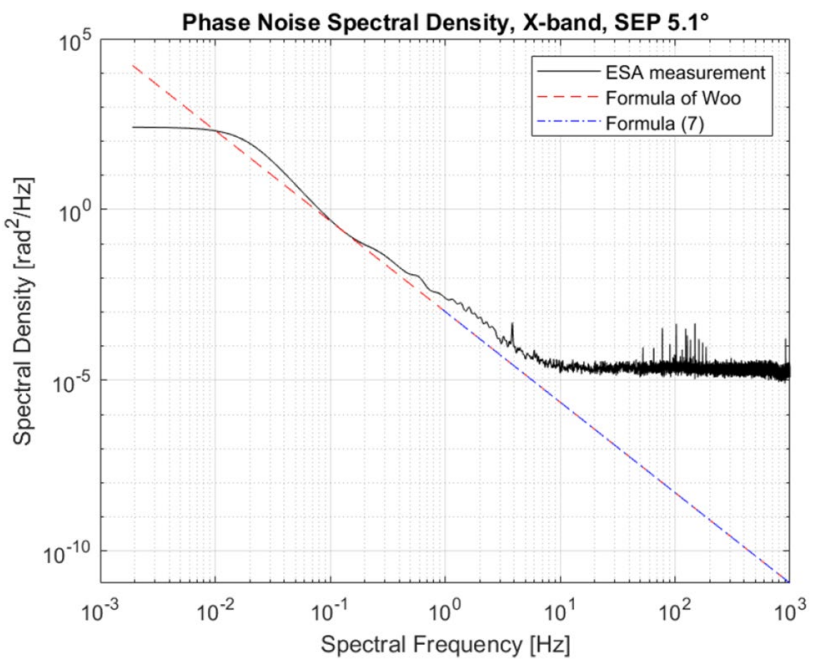

Fig. 6 Spectral density derived from the X-band measurement data with SEP $5.1^{\circ}$ of [7]. Noise floor measured $-44.8 \mathrm{~dB} / \mathrm{Hz}$ in Table 1

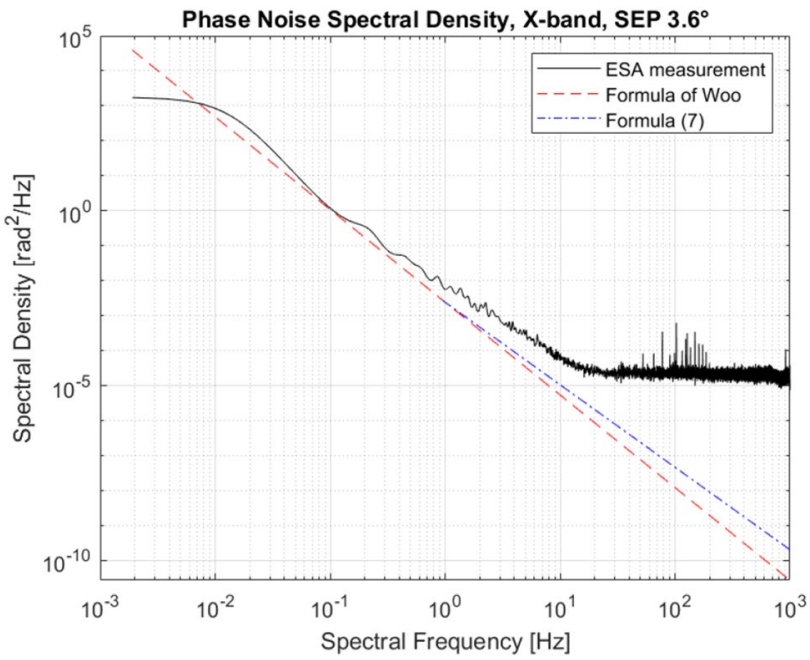

Fig. 7 Spectral density derived from the X-band measurement data with SEP $3.6^{\circ}$ of [7]. Noise floor measured $-45.0 \mathrm{~dB} / \mathrm{Hz}$ in Table 1

spectral densities for Ka-band correspondingly. It can be clearly recognized, that beyond the spectral frequency of about $10 \mathrm{~Hz}$, a noise floor with a level of about $-50 \mathrm{~dB} / \mathrm{Hz}$ prevails and dominates over the solar phase scintillation, also explained in the Eq. (5). This value of about $-50 \mathrm{~dB} /$ $\mathrm{Hz}$ corresponds to the received carrier to noise density ratio, as shown in Table 1.

The simulated phase scintillation spectral densities for $\mathrm{SEP}=1.2^{\circ}, 2.9^{\circ}, 3.6^{\circ}$, and $5.1^{\circ}$, using modified Woo's formula and by considering the scaling factor for the Kaband carrier frequencies, as well as the turn-around-factor $\mathrm{G}$ for the coherent two-way mode $\mathrm{X} / \mathrm{Ka}$, are displayed with dashed lines in the diagrams correspondingly.

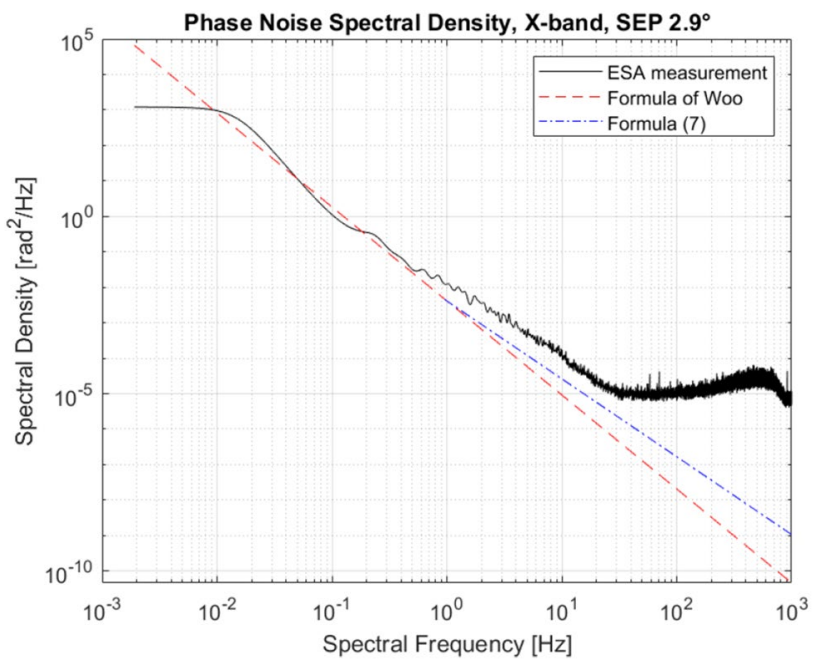

Fig. 8 Spectral density derived from the X-band measurement data with SEP $2.9^{\circ}$ of [7]. Noise floor measured $-51.3 \mathrm{~dB} / \mathrm{Hz}$ in Table 1

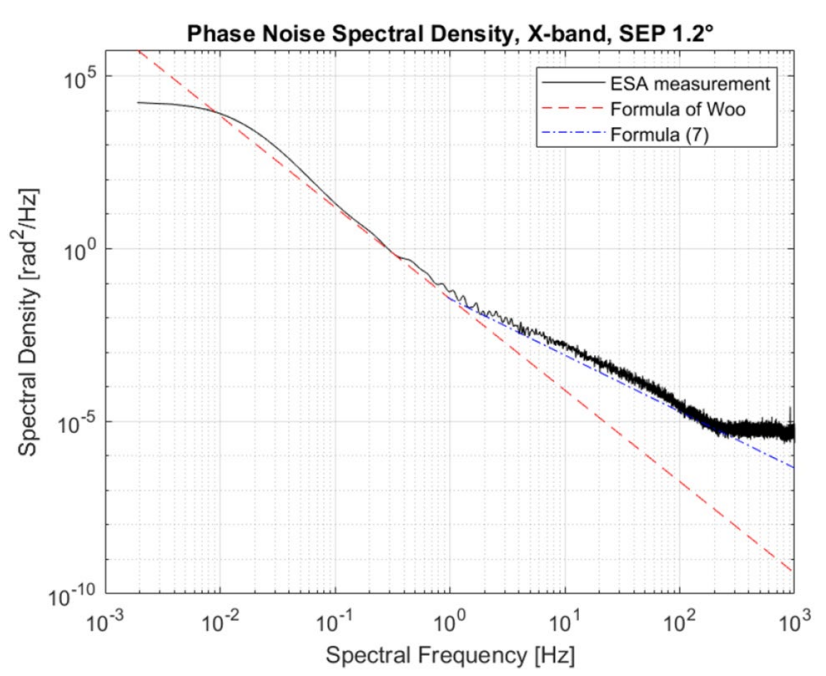

Fig. 9 Spectral density derived from the X-band measurement data with SEP $1.2^{\circ}$ of [7]. Noise floor measured $-48.9 \mathrm{~dB} / \mathrm{Hz}$ in Table 1

Table 1 BepiColombo superior solar conjunction measurement parameters

\begin{tabular}{lllll}
\hline Band & SEP $\left[{ }^{\circ}\right]$ & $\begin{array}{l}\text { Carrier power } \\
P_{\mathrm{c}}[\mathrm{dBm}]\end{array}$ & $\begin{array}{l}\text { Th. noise no } \\
{[\mathrm{dBm} / \mathrm{Hz}]}\end{array}$ & $\mathrm{No} / \mathrm{Pc}[\mathrm{dB} / \mathrm{Hz}]$ \\
\hline $\mathrm{X} / \mathrm{X}$ & 5.1 & -75.8 & -120.6 & -44.8 \\
$\mathrm{X} / \mathrm{X}$ & 3.6 & -75.8 & -120.8 & -45.0 \\
$\mathrm{X} / \mathrm{X}$ & 2.9 & -71.5 & -120.4 & -48.9 \\
$\mathrm{X} / \mathrm{X}$ & 1.2 & -66.9 & -118.2 & -51.3 \\
$\mathrm{X} / \mathrm{Ka}$ & 5.1 & -71.2 & -117.2 & -46.0 \\
$\mathrm{X} / \mathrm{Ka}$ & 3.6 & -71.3 & -116.9 & -45.6 \\
$\mathrm{X} / \mathrm{Ka}$ & 2.9 & -67.4 & -114.6 & -47.2 \\
$\mathrm{X} / \mathrm{Ka}$ & 1.2 & -71.8 & -116.8 & -45.0 \\
\hline
\end{tabular}




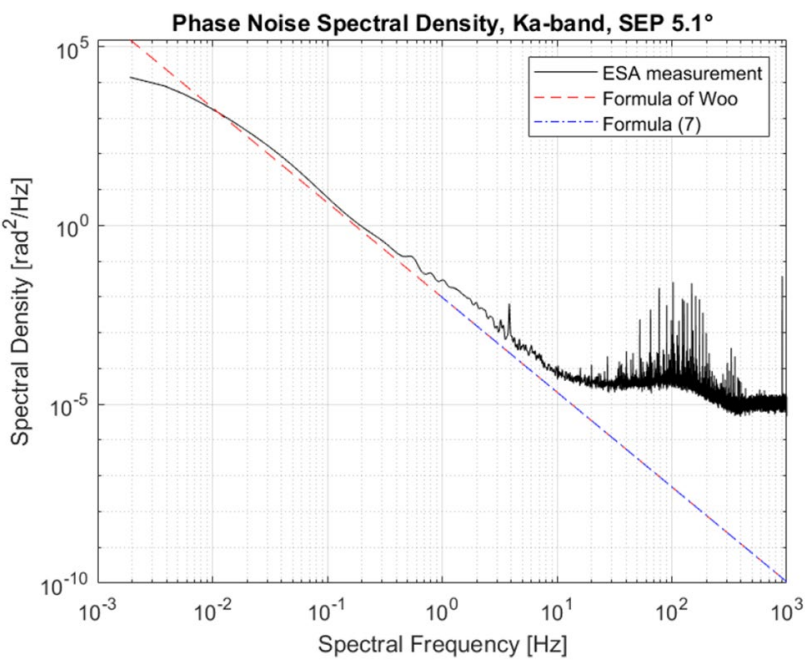

Fig. 10 Spectral density derived from the Ka-band measurement data with SEP $5.1^{\circ}$ of [7]. Noise floor measured $-46.0 \mathrm{~dB} / \mathrm{Hz}$ in Table 1

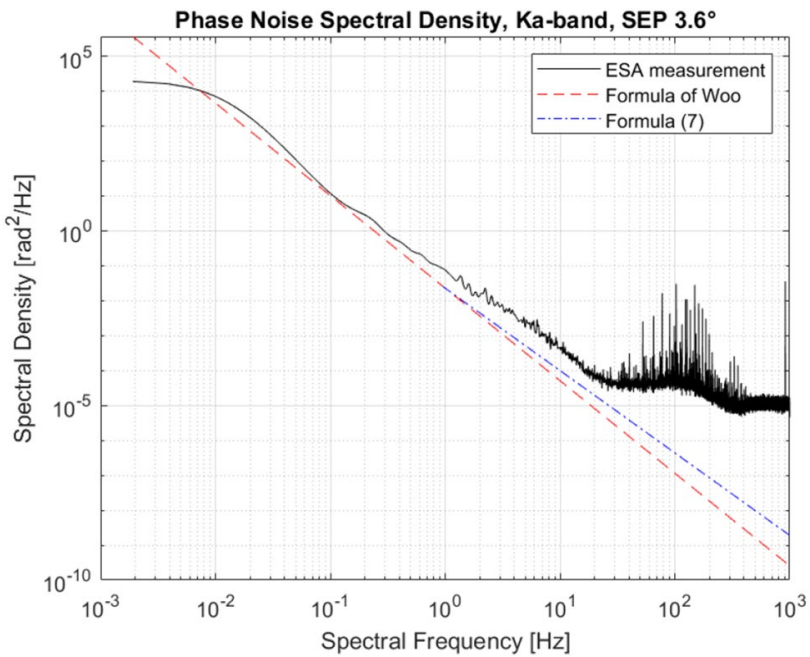

Fig. 11 Spectral density derived from the Ka-band measurement data with SEP $3.6^{\circ}$ of [7]. Noise floor measured $-45.0 \mathrm{~dB} / \mathrm{Hz}$ in Table 1

\section{Deviation of spectral density from the model for small SEP angles between $10^{\circ}-10^{2} \mathrm{~Hz}$}

It should be pointed out that the Figs. 6, 7, 8, 9, 10, 11, 12 and 13 present a deviation from the predicted slope at frequencies above approximately $1 \mathrm{~Hz}$, before the thermal noise floor becomes dominating. Woo already mentioned that the model could present deviations at frequencies above the characteristic fluctuation frequency associated with the Fresnel size, i.e. around $1 \mathrm{~Hz}$, especially

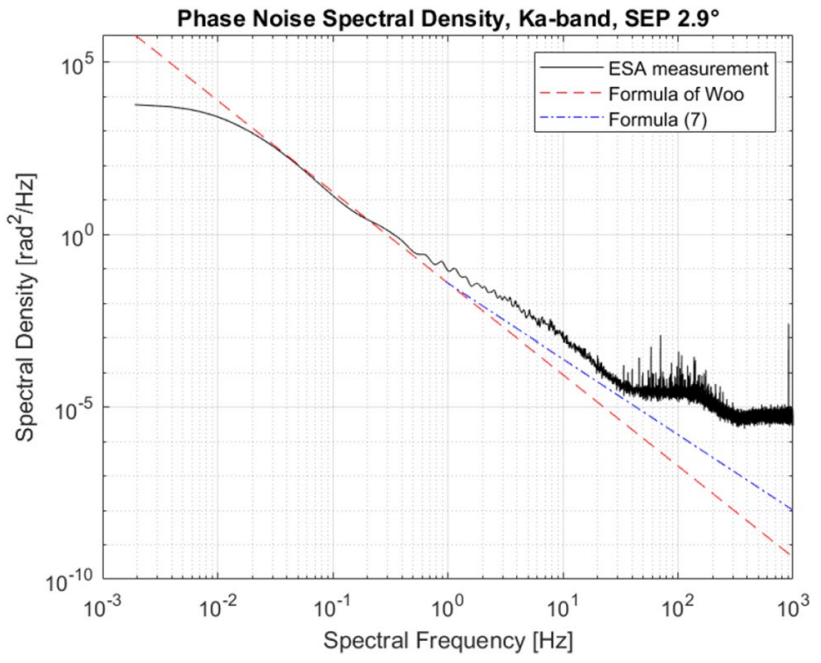

Fig. 12 Spectral density derived from the Ka-band measurement data with SEP $2.9^{\circ}$ of [7]. Noise floor measured $-45.0 \mathrm{~dB} / \mathrm{Hz}$ in Table 1

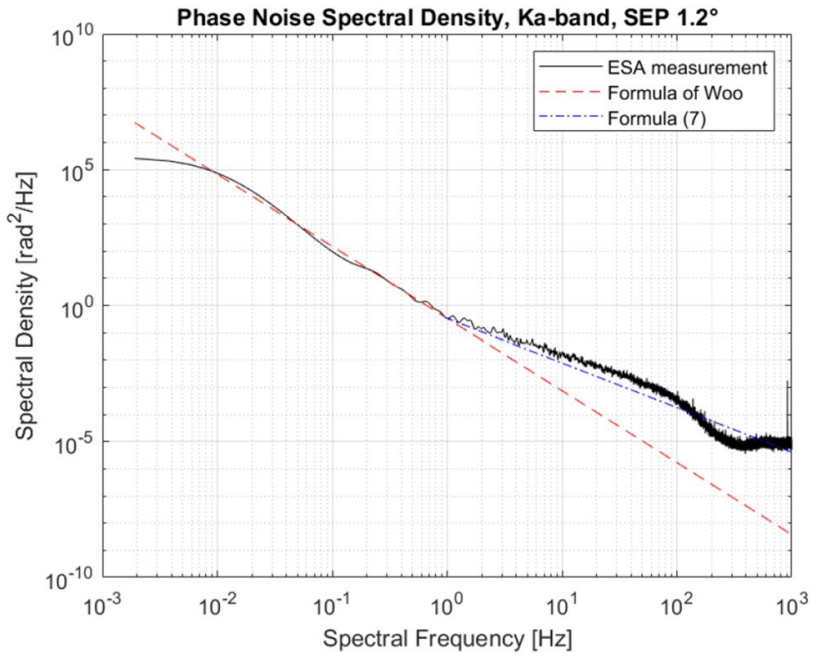

Fig. 13 Spectral density derived from the Ka-band measurement data with SEP $1.2^{\circ}$ of [7]. Noise floor measured $-47.2 \mathrm{~dB} / \mathrm{Hz}$ in Table 1

when the scintillation is stronger [1,3]. The cause for the second slope in the spectral density at small SEP angles would require further investigation and remains a primary goal for future work. One possible reason could be strong solar plasma activities above $1 \mathrm{~Hz}$. For the time being, we could consider an additional factor $\gamma=\left(\mathrm{SEP} / 5.1^{\circ}\right)^{1 / 3}$ (see the blue colored lines in Figs. 7, 8, 9, 11, 12, 13) which better approximates the model to the measured data: 


$$
S_{\Phi R}(f)=\left(\frac{2.3}{f_{c}}\right)^{2} \cdot 6.534 \cdot f^{-2.65 \cdot \gamma} \cdot\left(\frac{R}{R_{s}}\right)^{-2.45} \cdot\left(1+G^{4}\right)\left[\frac{\mathrm{rad}^{2}}{\mathrm{~Hz}}\right]
$$

\section{Conclusions}

The BepiColombo open loop data collected during the superior solar conjunction campaign in March 2021 have been used to compare the spectral power density of phase fluctuations of the signal versus the solar phase scintillation model presented by Woo [1-3] and modified to scale to a different carrier frequency and applicable to coherent operations. The relatively good match between the modified phase scintillation formulas for X-band and Ka-band at small SEP angles, i.e. from $5.1^{\circ}$ to $1.2^{\circ}$ for the radio frequency transmission confirms the suitability of these modified phase scintillation formulas for the verification of the measurements for future interplanetary missions. The phase noise spectral density for $\mathrm{X}$-band and Ka-band increases slightly beyond about $1 \mathrm{~Hz}$ for small SEP angles. This changed slope can be approximated by an additional factor in the phase scintillation formula. Further investigations are planned. On one hand these modified phase scintillation formulas can be used for future mission planning of $\mathrm{X}$-band and $\mathrm{Ka}$-band radio frequency communication links between the earth ground station and the spacecrafts, on the other hand measurement results of the phase scintillation can be used to observe the solar coronal ejections and prewarning the possible solar wind particles propagating to the earth.

Acknowledgements Shun-Ping Chen would like to thank the Department of Electrical Engineering and Information Technologies, Institute of Communication Technologies of Darmstadt University of Applied Sciences h-da, and European Space Agency, Operations Centre ESA/ ESOC for the valuable opportunity to be a visiting scientist at ESA/ ESOC. He also likes to thank his h-da colleague Prof. Dr. Heinz Schmiedel for very good cooperation and support, and Michael Chen for careful proofreading and many valuable suggestions to improve the text, figure and formula format. Both authors appreciate the weekly project meetings organized by the GSY section head Marco Lanucara, and thank for many effective discussions with him, his detailed suggestions, ideas and explanations which significantly improved the results of the investigations.

Funding Open Access funding enabled and organized by Projekt DEAL.

Open Access This article is licensed under a Creative Commons Attribution 4.0 International License, which permits use, sharing, adaptation, distribution and reproduction in any medium or format, as long as you give appropriate credit to the original author(s) and the source, provide a link to the Creative Commons licence, and indicate if changes were made. The images or other third party material in this article are included in the article's Creative Commons licence, unless indicated otherwise in a credit line to the material. If material is not included in the article's Creative Commons licence and your intended use is not permitted by statutory regulation or exceeds the permitted use, you will need to obtain permission directly from the copyright holder. To view a copy of this licence, visit http://creativecommons.org/licenses/by/4.0/.

\section{References}

1. Woo, R.: Multifrequency techniques for studying interplanetary scintillations. Astrophys J 201, 238-248 (1975)

2. Woo, R.: Radial dependence of solar wind properties deduced from Helios 1/2 and Pioneer 10/11 radio scattering observations. Astrophys J 219, 727-739 (1978)

3. Woo, R., Armstrong, J.W.: Spacecraft radio scattering observation of the power spectrum of electron density fluctuations in the solar wind. J Geophys Res 84, A12 (1979)

4. DSMS Telecommunications Link Design Handbook, 810-005, Rev. E, 34-m and 70-m Telemetry Reception (2003)

5. Gardner, F.. M.: Phaselock Techniques, third Wiley, New York (2005)

6. Lanucara, M.: Spacelink impairments by solar effects. ESA Document, Issue 2, Rev. 0. (2013)

7. Villalvilla, J.: Selected X-band and Ka-band solar plasma measurement data of ESA BepiColombo superior solar conjunction. E-Mails on June 10, 2021 and on July 30 (2021)

8. Matlab. https://www.mathworks.com 